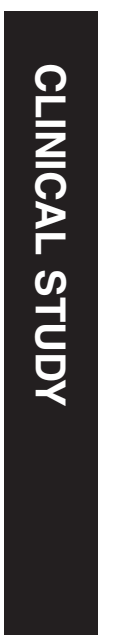

\title{
Outcomes of lower eyelid retractor recession and lateral horn lysis in lower eyelid elevation for facial nerve palsy
}

${ }^{1}$ Corneoplastic Unit, Queen Victoria Hospital NHS Foundation Trust, East Grinstead, UK

${ }^{2}$ Department of Ophthalmology, Ng Teng Fong General Hospital, National University Health System, Singapore

${ }^{3}$ Clinical Research Unit, $\mathrm{Ng}$ Teng Fong General Hospital, National University Health System, Singapore

Correspondence: R Malhotra, Corneoplastic Unit, Queen Victoria Hospital NHS Foundation Trust, Holtye Rd, East Grinstead RH19 3DZ, UK Tel: +44 (0)13 4241 4187; Fax: +44 (0)13 42414106 . E-mail: Raman.Malhotra@ qvh.nhs.uk

Received: 16 May 2016 Accepted in revised form: 2 July 2017

Published online:

1 September 2017

This paper has been previously presented at the American Society of Ophthalmic Plastic and Reconstructive Surgery, October 2016, Chicago, USA.

\begin{abstract}
Purpose To report outcomes and complications of lower eyelid retractor recession and lateral horn lysis (RR) for lower eyelid elevation in patients with facial nerve palsy (FNP).

Patients and methods Retrospective review. Patients with FNP undergoing RR alone (group 1) or with adjunctive procedures (canthal suspension-group 2, tarsorrhaphygroup 3, and full-thickness skin graft-group 4) during a 5-year period were included. Patient demographics, lagophthalmos, occurrence of eyelid malpositions, recurrent retraction, and repeat procedures were noted from medical records. Measures of lower eyelid height (LEH) and lid lag on downgaze were obtained from standard photographs. Results Forty-two patients (23 females, mean age was 59 years) were included. Mean follow-up was 24 months (range 6-77). Median improvement in LEH following surgery was significant in Group $1(0.90 \mathrm{~mm}$, IQR: $0.37-0.91, P=0.20$ ) and in Group 2 (0.51 mm, IQR: 0.30-1.37, $P<0.001$ ), respectively. Median improvement in blink lagophthalmos was $3.00 \mathrm{~mm}$ (IQR: 3.00-3.50, $P=0.02$ ) in Group 1 and $3.50 \mathrm{~mm}$ (IQR: 1.75$5.00, P<0.001)$ in Group 2. Median improvement in lagophthalmos on gentle eye closure was $2.00 \mathrm{~mm}$ (IQR: $1.50-3.00, P=0.02$ ) in Group 1 and $1.00 \mathrm{~mm}$ (IQR: 0-2.13, $P=0.006$ ) in Group 2. No new cases of ectropion were noted. $23.5 \%$ of patients required a repeat RR at a mean interval of 20 (range 1-70) months and a further $9.8 \%$ required a third procedure at a mean interval of 21 (range 18-29) months.

Conclusion RR improves lower eyelid retraction and lagophthalmos in FNP either alone or when combined as an adjunctive
\end{abstract}

P Tan ${ }^{1,2}$, J Wong ${ }^{3}$, WF Siah ${ }^{1}$ and R Malhotra ${ }^{1}$

procedure. It does not aggravate paralytic ectropion although repeated retractor recessions may be required to improve retraction.

Eye (2018) 32, 338-344; doi:10.1038/eye.2017.174; published online 1 September 2017

Introduction

Lower eyelid retraction and paralytic ectropion due to facial nerve palsy (FNP) contributes to lagophthalmos and sight-threatening exposure keratopathy. Many procedures have been described to address lower- eyelid retraction. These include tarsorrhaphy, ${ }^{1}$ canthal suspension, ${ }^{2,3}$ periocular placement of fascia lata slings ${ }^{4}$ and mid-facial sub-orbicularis oculi muscle/fat pad elevation. ${ }^{5-7}$ Lower eyelid retractor recession (RR) has also been previously described as a procedure for correction of lower eyelid retraction. ${ }^{8-11}$ The rationale for RR in FNP stems from the principles of upper eyelid levator recession, ${ }^{12,13}$ either with or without upper eyelid loading ${ }^{14-16}$ to improve lagophthalmos. Weakening of the pull by unopposed lower eyelid retractors by $\mathrm{RR}$ is analogous to weakening the levator to correct upper eyelid retraction and improve lagophthalmos.

However, long-term follow-up of postoperative outcomes of RR in patients with FNP, specifically the risks of occurrence of eyelid malpositions and lid lag on downgaze with disinsertion of the retractor complex remain unknown.

Our study aims to report the post-operative outcomes of RR (amount of lower eyelid elevation, improvement of lagophthalmos, incidence of eyelid malpositions, lid lag on downgaze, and need for repeat procedures) in patients with FNP. 


\section{Materials and methods}

We conducted a retrospective review of the medical records of all patients with FNP who underwent RR performed/supervised by a single surgeon (RM) during a 5-year period (January 2010-September 2015). Inclusion criteria were patients with FNP who underwent RR alone or in conjunction with other lower eyelid procedures including: lateral tarsal strip, lateral canthopexy, medial canthal tendon (MCT) plication and suture sling, ${ }^{17}$ medial/lateral tarsorrhaphy, or lower eyelid fullthickness skin grafts (FTSG). Patients with less than 3 months follow-up were excluded.

The surgical technique is as follows: A transconjunctival incision or a lateral canthotomy/cantholysis incision is first made. The lower eyelid retractors are then dissected from the conjunctiva posteriorly and the septum/inferior fat pads anteriorly with Westcott scissors. The lateral horns of the inferior retractors are lysed and the inferior retractors are now noted to be mobile and free. The inferior retractors are then simply replaced in the inferior fornix without suturing. The lower eyelid is noted at this point to be at a higher position as compared to before the surgery.

Patient demographics (age, gender) and clinical characteristics of FNP (etiology of FNP, laterality, amount of blink lagophthalmos, lagophthalmos on eye closure) were obtained from medical records. Post-operative complications of recurrence of lower eyelid retraction and occurrence of eyelid malpositions were noted. The number and nature of repeat RR procedures patients underwent over the 5-year follow-up period was reviewed.

We classified lower eyelid procedures as Group 1: patients who underwent RR and lateral horn lysis only. Group 2: RR and canthal support in the form of lateral canthopexy/ lateral tarsal strip/ MCT plication. Group 3: RR and tarsorrhaphy. Group 4: RR and FTSG.

On the basis of standard photographs, we reported the following post-operative outcome measures: (1) change in lower eyelid height; (2) subjective improvement in lower eyelid contour and lateral flare; and (3) lid lag on downgaze. All measurements were performed with the Photoshop measurement feature of Adobe Photoshop CC (San Jose, CA, USA) (Version 2015 16.1.2). The ruler tool was used to measure the various distances as detailed below to the nearest $0.1 \mathrm{~mm}$. An example of measurement of lower eyelid height is shown in Figure 1.

Lower eyelid height was calculated from standard photographs of patients in straight gaze by measuring the distance from the central pupil reflex to the central lower eyelid margin with the ruler tool of the Adobe Photoshop program. The measurement was then divided by the horizontal corneal diameter, which is taken to be the

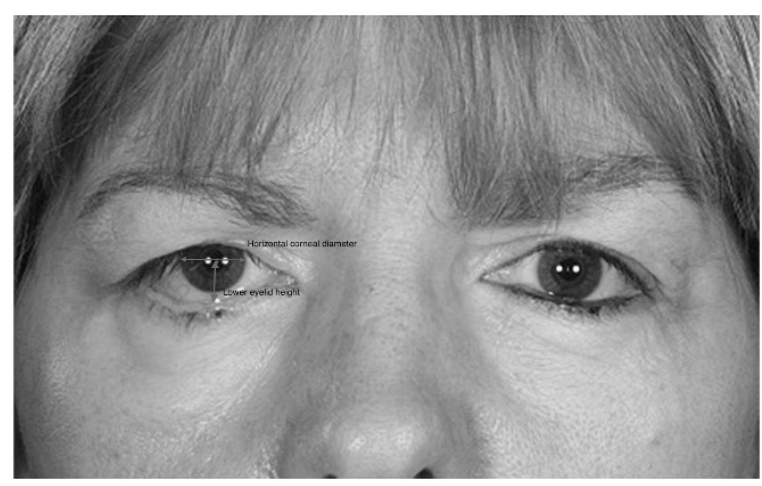

Figure 1 Example of measurement of lower eyelid height from standard photographs with Adobe Photoshop tool. The distance from the central pupil reflex to the central lower eyelid margin is measured and divided by the horizontal corneal diameter, which is taken to be the white-to-white distance on standard photographs. This ratio was then multiplied by a constant $(K=11.68)$ to give the central pupil to lower eyelid distance. A full colour version of this figure is available at the Eye journal online.

white-to-white distance on standard photographs. This ratio was then multiplied by a constant $(K=11.68)$ to give the central pupil to lower eyelid distance. We derived the constant $(K)$ from Hashemi et al, ${ }^{18}$ who reviewed 410 patients by using the Orbscan II and found the mean corneal diameter to be $11.68 \mathrm{~mm}$.

Lower eyelid lateral flare was compared with the contralateral eye and was recorded before and after surgery as being absent, subtle or significant.

Lid lag of the lower- eyelid on downgaze was compared to the contralateral eye before and after surgery on standard photographs and graded as being no change, improved, or worsened.

All photographs were taken by Nikon D700 camera (Nikon, Tokyo, Japan) with a $105 \mathrm{~mm}$ lens with a shutter speed of $1 / 125 \mathrm{~s}$, and the aperture was $\mathrm{f} / 20$. All photographs were taken at a standard distance $(1.5 \mathrm{~m})$ with standard lighting with a flashlight set at a $45^{\circ}$ angle.

Queen Victoria Hospital NHS Foundation Trust Institutional Review Board approval was obtained for this retrospective audit. The study adhered to the tenets of the Declaration of Helsinki.

\section{Statistical analysis}

Descriptive statistics would be reported as mean and SD for parametric data and as median (interquartile rangeIQR in short) for nonparametric data. Parametric tests would be used if there were no major violations of the normality assumption, otherwise nonparametric tests would be used. Hence, paired $t$-test or Wilcoxon SignedRank test would be used to compare paired nonparametric pre- and post-operative data for lower eyelid height and lagophthalmos for Groups 1 and 2, 
respectively. Thereafter, two-sample $t$-test or MannWhitney test was used to compare the overall change in clinical parameters following surgery between Groups 1 and 2. We omitted Groups 3 and 4 from the analysis of pre- and post-operative changes of the above as concurrent tarsorrhaphy or FTSG would have a confounding effect on eyelid height and lagophthalmos. All statistical analyses were performed using the statistical software SPSS (IBM, Armonk, NY, USA, 2015). A $P$-value of $<0.05$ was considered statistically significant.

\section{Results}

Fifty-one patients with FNP underwent RR either singly or in conjunction with other procedures over the 5-year period. Nine patients were excluded due to follow-up of less than 6 months. A total of 42 patients (23 females) were analyzed.

Mean age was $58.9(\mathrm{SD}=18.8)$ years; mean follow-up period was $23.8(\mathrm{SD}=15.4)$ months. Clinical characteristics of FNP and the types of lower eyelid procedure performed are shown in Table 1. The most common cause of FNP was acoustic neuroma.

Lower eyelid procedures included group 1: RR and lateral horn lysis only $(n=8)$, group 2: RR and canthal support in the form of lateral canthopexy/ lateral tarsal strip/ MCT plication $(n=27)$, group 3: RR and tarsorrhaphy $(n=1)$ and group 4: RR and FTSG $(n=6)$.

No patients had development of new-onset lower eyelid malpositions. Four patients were noted to have postoperative lower eyelid ectropion within 6 months after their first surgeries. Of these, three patients had pre-existing lower eyelid ectropion, which was not completely corrected after surgery. An example of a patient is shown in Figures $2 \mathrm{a}-\mathrm{d}$. This patient had improvement of the left lower eyelid ectropion after her first surgery of RR and canthal suspension (Figure $2 b$ ); however, recurrence of ectropion was noted 6 months after the first surgery (Figure 2c). She went on to undergo a repeat RR and FTSG with improvement of her lower eyelid ectropion (Figure 2d).

Another patient without any pre-existing eyelid malpositions (Figure 2e) developed left lower eyelid cellulitis 2 days after RR and canthal suspension surgery (Figure 2f). Although the cellulitis responded well to intravenous antibiotics, the patient developed punctal ectropion as a result (Figures $2 g$ and $h$ ). He was asymptomatic and no further surgical intervention was required for him.

\section{Lower eyelid height}

Data for lower eyelid height were analyzed using nonparametric approach as there were violations on the normality assumption of the data. Median improvement
Table 1 Clinical characteristics of 42 patients with Facial nerve palsy

\begin{tabular}{|c|c|}
\hline Clinical characteristic & $\mathrm{N}(\%)$ \\
\hline \multicolumn{2}{|l|}{ Laterality } \\
\hline Right & $23(55)$ \\
\hline Left & $19(45)$ \\
\hline \multicolumn{2}{|l|}{ Etiology of Facial nerve palsy } \\
\hline Acoustic neuroma & $13(31)$ \\
\hline Bell's Palsy & $6(14)$ \\
\hline Congenital & $5(12)$ \\
\hline Congenital choleasteatoma & $1(2)$ \\
\hline Intracranial tumors & $6(14)$ \\
\hline Malignant otitis externa & $1(2)$ \\
\hline Parotid tumor & $3(7)$ \\
\hline Trauma & $2(5)$ \\
\hline Vestibular schwannoma & $5(12)$ \\
\hline \multicolumn{2}{|l|}{ Primary surgical procedures on lower eyelid $(\mathrm{n}=42)$} \\
\hline Group 1: Retractor recession only & $8(19)$ \\
\hline Group 2: Retractor recession and canthal support ${ }^{a}$ & $27(64)$ \\
\hline Group 3: Retractor recession and tarsorrhaphy & $1(2)$ \\
\hline $\begin{array}{l}\text { Group 4: Retractor recession and full-thickness skin } \\
\text { graft }\end{array}$ & $6(14)$ \\
\hline \multicolumn{2}{|l|}{ Second repeat procedures on lower eyelid $(\mathrm{n}=12)$} \\
\hline Retractor recession only & $0(0)$ \\
\hline Retractor recession and canthal support ${ }^{a}$ & $8(67)$ \\
\hline Retractor recession and tarsorrhaphy & $1(8)$ \\
\hline Retractor recession and full-thickness skin graft & $3(25)$ \\
\hline \multicolumn{2}{|l|}{ Third repeat procedures on lower eyelid $(\mathrm{n}=5)$} \\
\hline Retractor recession only & $1(20)$ \\
\hline Retractor recession and canthal support ${ }^{a}$ & $1(20)$ \\
\hline Retractor recession and tarsorrhaphy & $1(20)$ \\
\hline Retractor recession and full-thickness skin graft & $2(40)$ \\
\hline
\end{tabular}

in lower eyelid height was $0.90 \mathrm{~mm}$ (IQR: 0.37-0.91) in Group 1 and $0.51 \mathrm{~mm}$ (IQR: 0.30-1.37) in Group 2, respectively. Wilcoxon Signed-Rank test showed significant improvement in lower eyelid height in both groups following surgery (Group 1: $P=0.20$; Group 2: $P<0.001)$. There was no significant difference between the two procedures in terms of magnitude of improvement $(P=0.92)$.

\section{Lagophthalmos}

Similarly, data for lagophthalmos were analyzed using nonparametric approach as there were violations on the normality assumption. Median improvement in blink lagophthalmos was $3.00 \mathrm{~mm}$ (IQR: 3.00-3.50) in Group 1 and $3.50 \mathrm{~mm}$ (IQR: $1.75-5.00$ ) in Group 2. There was significant improvement in blink lagophthalmos in both groups (Group 1: $P=0.02$; Group 2: $P<0.001$ ) but the difference between the groups was not statistically 
a
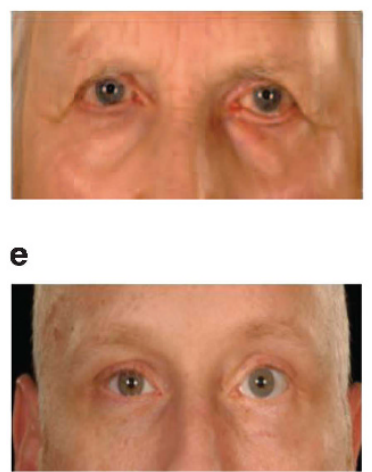

b

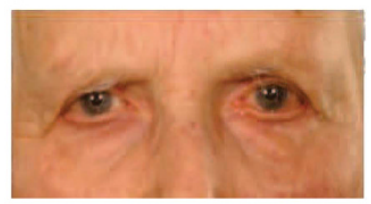

f

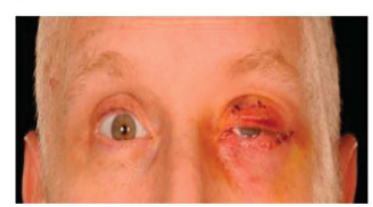

c

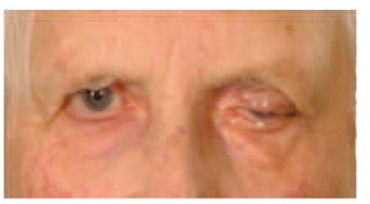

g

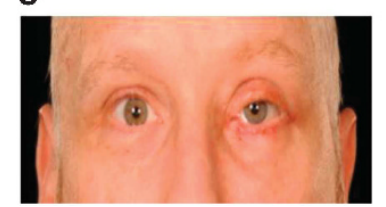

d

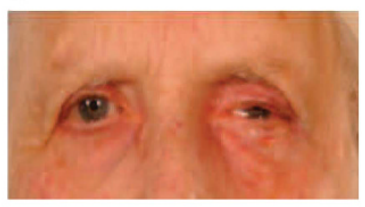

h

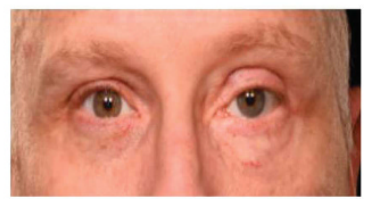

Figure 2 Lower eyelid ectropion. (a) Left lower eyelid ectropion pre-operatively. (b) post-operatively three months after retractor recession and canthal suspension procedure (c) recurrence of lower eyelid ectropion six months after first surgery (d) patient underwent a second procedure of retractor recession and full-thickness skin graft with improvement of ectropion post-operatively. (e) Another patient with left facial nerve palsy without lower eyelid malposition (f) Left lower eyelid cellulitis after retractor recession and canthal suspension surgery (g) Resolution of cellulitis with intravenous antibiotics. (h) Residual left punctal ectropion 8 months after surgery.

a

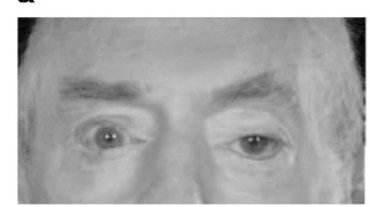

c

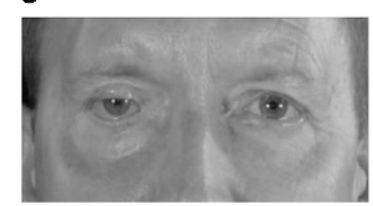

b

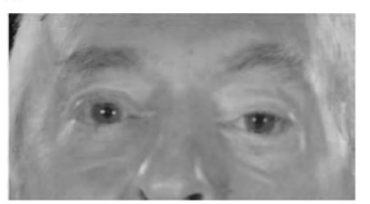

d

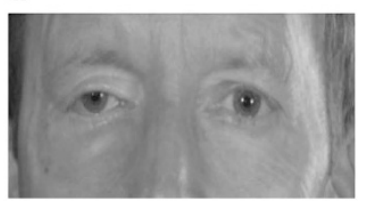

Figure 3 Changes in lower eyelid contour and lateral flare before and after surgery. (a) Left lower eyelid lateral flare preoperatively (b) post-operatively. (c) Right lower eyelid lateral flare pre-operatively (d) post-operatively. A full colour version of this figure is available at the Eye journal online.

significant $(P=0.98)$. Median improvement in lagophthalmos on gentle eye closure was $2.00 \mathrm{~mm}$ (IQR: 1.50-3.00) in Group 1 and $1.00 \mathrm{~mm}$ (IQR: 0-2.13) in Group 2. Significant improvements in gentle eye closure were seen in both groups (Group 1: $P=0.02$; Group 2: $P=0.006$ ) but the difference between the groups was not statistically significant $(P=0.15)$. Median change in lagophthalmos on forced eye closure was zero for each group (Group $1 \mathrm{IQR}$ : 0-1.50; Group 2 IQR: 0-1.13). There was no evidence of improvement on forced eye closure significantly for both groups (Group 1: $P=0.35$; Group 2: $P=0.09$ ) and between groups $(P=0.74)$.

\section{Lower eyelid contour including lateral flare}

Figure 3 depicts the pre- and post-operative changes in lower eyelid flare for some patients in the study. $29 \%$ of

a

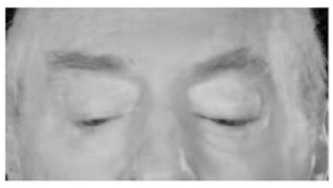

c

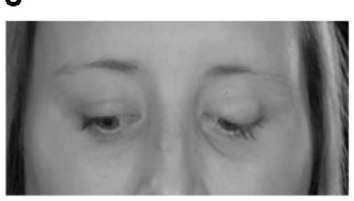

b

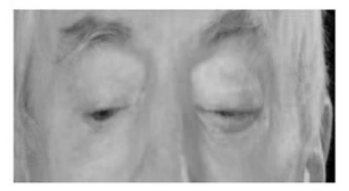

d

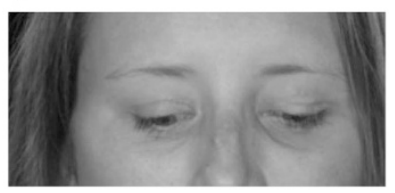

Figure 4 Lid lag on downgaze. (a) No lid lag on left eye preoperatively. (b) Lid lag present on left eye post-operatively. (c) No lid lag on left eye pre-operatively. (d) Lid lag present on left eye post-operatively. A full colour version of this figure is available at the Eye journal online.

patients had improvement in lower eyelid contour and lateral flare. Figures 3a-d demonstrates two examples of patients with improvement in lower eyelid lateral flare after surgery. Two patients had worsening of lateral flare. Three patients had evidence of preoperative lower eyelid lateral flare, which was unchanged after surgery.

\section{Worsening of lid lag on downgaze}

Four patients had pre-existing lid lag on downgaze, which was unchanged after surgery. Eleven patients were noted to have new-onset lid lag on downgaze postoperatively, examples as shown in Figure 4. These patients were contacted via telephone interview with regards to ipsilateral impairment of inferior visual field on downgaze due to lid lag. One patient reported slight impairment of inferior field of vision due to lid lag. 


\section{Number and type of repeat retractor recession procedures performed}

Twelve patients (28.6\%) underwent a second procedure and five patients $(11.9 \%)$ underwent a third procedure in the follow-up period. The indication for repeat procedures was recurrence of lower eyelid retraction and exposure keratopathy in all cases. The mean time to recurrence of lower eyelid retraction was 20 months (range 1-70) The types of repeat procedures performed are detailed in Table 1. Lower eyelid elevation improved further by $1.25 \mathrm{~mm}$ for eight patients. Of the four patients with no improvement in lower eyelid elevation, two patients had undergone RR and MCT plication and suture sling procedures and another two patients underwent $R R$ and FTSG. These four patients went on to undergo a third procedure. A fifth patient went on to have a third procedure as well due to recurrence of lower eyelid retraction 22 months after the second procedure. Of note, two patients who also had recurrence of lower eyelid retraction after the first procedure declined further surgical intervention.

\section{Discussion}

Lower eyelid retraction in patients with FNP poses a surgical challenge as various factors may be involved. ${ }^{19,20}$ These include orbicularis oculi weakness giving rise to an atonic lower eyelid, tension on the anterior lamellar of the lower eyelid from mid face descent, skin contracture ${ }^{21}$ in patients with FNP, and unopposed action of the lower eyelid retractors. Our study shows that RR alone or as an adjunct to other procedures is an effective adjunct for lower eyelid elevation even in the context of paralytic ectropion. Based upon our long-term follow-up, RR does not worsen eyelid malpositions or cause significant inferior visual field limitation on downgaze. Due to the ongoing disease process of FNP, lower eyelid retraction can be recurrent and repeat RR can be safely performed with further improvement in lower eyelid height and lagophthalmos.

The lower eyelid retractors consist of two layers, ${ }^{22}$ the posterior layer being the thicker of the two includes smooth muscle fibers and reaches forward to the anterior, inferior and posterior surface of the lower tarsal plate. The main role of this posterior layer is to pull the eyelid inferoposteriorly. The unopposed pull of the lower eyelid retractors with weakened orbicularis oculi muscles in FNP results in lower eyelid retraction.

Lower eyelid RR has been reported as early as 1990 by Holds et $\mathrm{al}^{8}$ who described a minimal incision 'en-glove' retractor lysis of the lower eyelid in patients with lower eyelid retraction post blepharoplasty or trauma and achieving up to $3 \mathrm{~mm}$ improvement in inferior scleral show. Recession of the posterior layers of the retractors (as well as severing medial/lateral horns) has also been described as effective maneuvers to help address cicatricial eyelid entropion ${ }^{23}$ and following inferior rectus recession ${ }^{24}$ to prevent eyelid malposition. Subsequent studies reported the effectiveness of RR in management for FNP. Compton et al performed recession and extirpation of lower eyelid retractors in 11 patients with FNP. ${ }^{9}$ They reported an improvement of $3.0 \mathrm{~mm}$ in lower eyelid height and $4.8 \mathrm{~mm}$ of lagophthalmos. However their follow-up period was relatively short (mean of 15.6 weeks). All patients also had concurrent upper eyelid loading with gold weight placement, which would confound post-operative lagophthalmos measurements. The distance from the corneal light reflex to the lower eyelid (MRD2) was used as the outcome measurement of lower eyelid height. However, no mention of standard photographs or magnifications was specified in the study. As such measurement errors may arise if different magnifications of photographs for each patient are used. Yoo $e t a l^{10}$ reported their technique of a minimally invasive, orbicularis sparing (MIOS) trans-conjunctival retractor release and lateral horn lysis. Their series included 11 patients with FNP. MIOS alone achieved $1.40 \mathrm{~mm}$ correction in five patients; six patients with adjunctive tarsorrhaphy achieved $2.40 \mathrm{~mm}$ correction. Their study has the strengths of a longer follow-up period (mean 11, range 6-21 months) than that of Holds et al. ${ }^{8}$ The same examiner also measured the lower eyelid height clinically at presentation and at the final follow-up.

Our study is a larger series of patients with a longer follow-up period and results that are similar to Yoo et al. $\mathrm{RR}$ alone (Group 1) achieved $0.90 \mathrm{~mm}$ improvement of lower eyelid height, whilst RR and canthal support procedures (Group 2) achieved $0.51 \mathrm{~mm}$ improvement in lower eyelid height. RR alone or with canthal support procedures also resulted in significant improvements in blink lagophthalmos and lagophthalmos on gentle closure. There was, however, no significant difference between the two groups for all the parameters measured. One may have assumed that additional canthal support procedures would result in more significant improvements in lower eyelid heights/inferior scleral show or lagophthalmos, however, being a retrospective review, patients selected for these procedures would not have been similar. Canthal support in group 2 would have been deemed necessary for this group (ie, possibly more severe eyelid retraction or concurrent ectropion, inferior scleral show, lagophthalmos) and therefore, it is difficult to extrapolate any true comparison in outcomes of RR between the two groups. Selection of RR alone was certainly not purely based upon a younger age group as there was no significant difference in age $(P=0.29)$ between groups $1($ mean $=50.5, \mathrm{SD}=18.4)$ and 2 $($ mean $=58.0, \mathrm{SD}=16.9)$. 
We excluded patients who had adjunctive tarsorrhaphy or FTSG from the analysis as these additional procedures could possible confound the results and have an additive effect to lower eyelid elevation. This proved to be true when we analyzed the post-operative outcomes in Group 4 patients. The median improvement (IQR) in lower eyelid height, blink lagophthalmos, lagophthalmos on gentle eye closure and lagophthalmos on forced eye closure was $1.16 \mathrm{~mm}$ (IQR: 0.51-3.09), $3.00 \mathrm{~mm}$ (IQR: 2.00-6.00), $2.00 \mathrm{~mm}$ (IQR: 1.00-6.00) and $2.00 \mathrm{~mm}$ (IQR: 1.00-3.00) for Group 4 (FTSG). Although the improvement in lower eyelid height and inferior scleral show is superior to that of RR alone or RR and canthal procedure, one should not rush into performing FTSG on every patient with lower eyelid RR. Instead, it is important that the correct procedure is individualized for the patient. In patients with mild lower eyelid retraction, RR may be an adequate procedure alone. However if other factors are present, these will then have to be addressed. For example, a patient with lower eyelid retraction and paralytic medial/lateral ectropion will benefit from adjunctive canthal suspension procedures or tarsorrhaphy. This also holds true for patients with anterior lamellar shortening-a FTSG would be necessary to correct adequately the amount of retraction. One must nevertheless bear in mind that a FTSG or tarsorrhaphy may be esthetically unacceptable to the patient. Careful consideration of the surgical pathology, extent of exposure keratopathy and patient's wishes is therefore imperative to achieve a good post-operative outcome.

It has been theorized that lower eyelid retractors disinsertion/involutional change can lead to anteriorposterior instability of the lower eyelid, which can contribute to ectropion or entropion. ${ }^{25,26}$ Our study found that RR alone or as an adjunct does not cause or aggravate eyelid malpositions. Four patients who had pre-existing paralytic ectropion did not have worsening of their ectropion. No new cases of ectropion occurred during our long follow-up period. This is similar to the results from Holds et al, Compton et al and Yoo et al. They had no complications of eyelid malpositions in their series. We believe that concurrent canthal support procedures in our study were beneficial in preventing the development of new-onset eyelid malpositions or aggravation of existing ones. Canthal support procedures are also effective in treatment of horizontal eyelid laxity, which contributes to lower eyelid retraction.

Although 11 patients were judged to have lid lag on downgaze post-operatively from photographs, only one patient mentioned a slight impairment to inferior visual field, not affecting activities of daily living. Yoo et al reported no reduced eyelid depression, inferior field limitation or problems reading in their study. However their method of assessment (clinical examination) differs from ours (standard photograph). The advantage of using photographs allows the grader to set a reference point to compare both eyes.

This paper reports long-term outcomes including the need for repeat RR procedures with further objective improvement in lower eyelid elevation. FNP is a disease that will have ongoing chronic changes to the eyelid and mid face. It is not surprising that some patients in our series developed recurrent retraction. RR alone in this group of patients however would not address adequately the amount of retraction, and adjunctive procedures may have to be considered. Anecdotally, we notice that in patients with previous RR, the recessed lower eyelid retractors can often still be found adherent to the underlying conjunctiva albeit at a lower position. There is therefore a role for repeat RR and lateral horn lysis and possibly the role for retractor extirpation.

Our study is limited by its retrospective nature and possible referral bias as our unit is a tertiary referral center for FNP. There is also no standardization of adjunctive surgeries, and one could argue that the additional procedures will themselves affect lower eyelid height or occurrence of eyelid malpositions and lid lag. However, this study is reflective of clinical practice, and it is difficult to be overly prescriptive based upon these results.

The strengths of our study include our long follow-up period, large study patient group and objective measurements of post-operative outcomes. Standardized photographs following a set protocol are mandated for all our FNP patients. Calculation of lower eyelid heights with a corneal constant ensures that measurements would not be affected by magnifications of photographs.

In conclusion, RR either alone or as an adjunctive procedure significantly improves lower eyelid height and lagophthalmos in patients with FNP while remaining safe. This procedure should therefore be considered when dealing with lower eyelid retraction in patients with FNP even in the presence of ectropion.

\section{Summary}

What was known before

- Lower eyelid retraction and paralytic lagophthalmos contributes to exposure keratopathy in patients with facial nerve palsy. Lower eyelid retractor recession has already been described as an effective procedure for lower eyelid elevation.

\section{What this study adds}

- Long-term follow-up with regards to complications like occurrence of lid malpositions, lid lag on downgaze and need for repeat procedures after lower eyelid retractor recession is lacking. Our study aims to report our experience with lower eyelid retractor recession in terms of improvement of lower eyelid height and incidence of the above-mentioned complications. 


\section{Conflict of interest}

The authors declare no conflict of interest.

\section{Acknowledgements}

We acknowledge Dr Garett Hellenthal for his assistance in statistical analysis.

\section{References}

1 Chang L, Olver J. A useful augmented lateral tarsal strip tarsorrhaphy for paralytic ectropion. Ophthalmology 2006; 113(1): 84-91.

2 Jordan DR, Anderson RL. The lateral tarsal strip revisited. The enhanced tarsal strip. Arch Ophthalmol 1989; 107(4): 604-606.

3 Fante RG, Elner VM. Transcaruncular approach to medial canthal tendon plication for lower eyelid laxity. Ophthal Plast Reconstr Surg 2001; 17(1): 16-27.

4 Sendul SY, Cagatay HH, Dirim B, Demir M, Acar Z, Olgun A et al. Effectiveness of the lower eyelid suspension using fascia lata graft for the treatment of lagophthalmos due to facial paralysis. Biomed Res Int 2015; 2015: 759793.

5 Elner VM, Mauffray RO, Fante RG, Harris M, Morton AD, Hassan AS. Comprehensive midfacial elevation for ocular complications of facial nerve palsy. Arch facial plast surg 2003; 5: 427-433.

6 Olver J. Raising the suborbicularis oculi fat (SOOF): its role in chronic facial palsy. Br J Ophthalmol 2000; 84: 1401-1406.

7 Alford EL. The SOOF lift as an adjunct in rehabilitation of facial paralysis: help or hype? Facial Plast Surg 2000; 16(4): 345-349.

8 Holds JB, Anderson RL, Thiese S. Lower eyelid retraction: a minimal incision surgical approach to retractor lysis. Ophthalmic Surg 1990; 21: 11.

9 Compton CJ, Clark JD, Nunery WR, Lee HB. Recession and extirpation of the lower eyelid retractors for paralytic lagophthalmos. Ophthal Plast Reconstr Surg 2015; 31(4): 323-324.

10 Yoo DB, Griffin GR, Azizzadeh B, Massry GG. The minimally invasive, orbicularis-sparing, lower eyelid recession for mild to moderate lower eyelid retraction with reduced orbicularis strength. JAMA Facial Plast Surg 2014; 16(2): 140-146.

11 Norris JH, Malhotra R. Composite Septo-retractor recession; a surigical technique for lower -eyelid retraction and review of the literature. Ophthal Plast Reconstr Surg 2011; 27: $447-452$.

12 Guillou-Jamard MR, Labbé D, Bardot J, Benateau H. Paul Tessier's technique in the treatment of paralytic lagophthalmos by lengthening of the levator muscle: evaluation of 29 cases. Ann Plast Surg 2011; 67(6): S31-S35.

13 Tessier P, Delbet JP, Pastoriza J, Lekiefre M. Paralized eyelids. Ann Chir Plast 1969; 14: 215-223.

14 Caesar RH, Friebel J, McNab AA. Upper lid loading with gold weights in paralytic lagophthalmos: a modified technique to maximize the long-term functional and cosmetic success. Orbit 2004; 23(1): 27-32.

15 Mavrikakis I, Malhotra R. Techniques for upper eyelid loading. Ophthal Plast Reconstr Surg 2006; 22(5): 325-330.

16 Bladen JC, Norris JH, Malhotra R. Indications and outcomes for revision of gold weight implants in upper eyelid loading. Br J Ophthalmol 2012; 96: 485-489.

17 Sira M, Norris JH, Nduka C, Malhotra R. Transcaruncular medial canthal tendon plication with lower eyelid suture sling in facial nerve palsy. Orbit 2014; 33(3): 159-163.

18 Hashemi H, KhabazKhoob M, Yazdani K, Mehravaran S, Mohammad K, Fotouhi A. White-to-white corneal diameter in the Tehran Eye Study. Cornea 2010; 29(1): 9-12.

19 Joseph SS, Joseph AW, Douglas RS, Massry GG. Periocular reconstruction in patients with facial paralysis. Otolaryngol Clin North Am 2016; 49(2): 475-872.

20 Seiff SR, Chang JS. The surgical management of ophthalmic complications of facial nerve palsy. Ophthal Plast Reconst Surg 1993; 9: 241-249.

21 Ziahosseini K, Venables V, Neville C, Nduka C, Patel B, Malhotra R. Occurrence and severity of upper eyelid skin contracture in facial nerve palsy. Eye (Lond) 2016; 30: 713-717.

22 Kakizaki H, Malhotra R, Madge SN, Selva D. Lower eyelid anatomy: an update. Ann Plast Surg 2009; 63(3): 344-351.

23 Kakizaki H, Zako M, Iwaki M. Lower eyelid lengthening surgery targeting the posterior layer of the lower eyelid retractors via a transcutaneous approach. Clin Ophthalmol 2007; 1: 141-147.

24 Kim DB, Meyer DR, Simon JW. Retractor lysis as prophylaxis for lower lid retraction following inferior rectus recession. J Pediatr Ophthalmol Strabismus 2002; 39: 198-202.

25 Putterman AM. Ectropion of the lower eyelid secondary to Muller's muscle-capsulopalpebral fascia detachment. Am J Ophthalmol 1978; 85(6): 814-817.

26 Wesley RE. Tarsal ectropion from detachment of the lower eyelid retractors. Am J Ophthalmol 1982; 93: 491-495. 